\author{
Danielle S. Ross, PhD, MSc, W. June Holstrum, PhD, \\ Marcus Gaffney, MPH, Denise Green, MPH, Robert F. Oyler, PhD, \\ and Judith S. Gravel, PhD
}

More than $90 \%$ of newborns in the United States are now being screened for hearing loss. A large fraction of cases of unilateral hearing loss and mild bilateral hearing loss are not currently identified through newborn hearing screening. This is of concern because a preponderance of research has demonstrated that unilateral hearing loss and mild bilateral hearing loss can lead to developmental delays and educational problems for some children. To help address this probable underidentification of unilateral hearing loss and mild bilateral hearing loss among infants and children, the Centers for Disease Control and Prevention Early Hearing Detection and Intervention program and the Marion Downs Hearing Center convened a workshop in Breckenridge, Colorado, in July 2005. During this workshop, several issues related to screening and diagnosing unilateral hearing loss and mild bilateral hearing loss were identified, as well as recommendations for future research in this area. Issues identified included the lack of standardized definitions for permanent unilateral hearing loss and mild bilateral hearing loss; the use of screening protocols that are primarily designed to identify bilateral and unilateral hearing losses of a moderate degree or greater (eg, above $40 \mathrm{~dB}$ ); calibration of screening equipment; availability of facilities that can provide the full range of audiologic, diagnostic, and management services to this pediatric population; and an overall lack of awareness by many professionals and families about the potential effect of unilateral hearing loss and mild bilateral hearing loss. Suggestions for future research, such as identifying ways to improve the identification of cases of unilateral hearing loss and mild bilateral hearing loss, were also discussed.

Keywords: mild bilateral hearing loss (MBHL); unilateral hearing loss (UHL); minimal hearing loss; newborn hearing screening; early hearing detection and intervention
$\mathrm{M}$ ore than $90 \%$ of newborns in the United States are now being screened for hearing loss. ${ }^{1}$ Despite this widespread screening coverage,

From Centers for Disease Control and Prevention, Atlanta, Georgia (DSR, MG, DG); McKing Consulting Corporation (WJH); Mississippi University for Women, Columbus (RFO); and Children's Hospital of Philadelphia, Pennsylvania (JSG).

The findings and conclusions in this article are those of the authors and do not necessarily represent the views of the Centers for Disease Control and Prevention.

Address correspondence to: Danielle S. Ross, PhD, MSc, Early Hearing Detection \& Intervention Program, Division of Human Development and Disabilities, National Center on Birth Defects \& Developmental Disabilities, Centers for Disease Control and Prevention, 1600 Clifton Road NE, Mailstop E-88, Atlanta, GA 30333; e-mail: dross3@cdc.gov. data indicate not all cases of unilateral hearing loss (UHL) and mild bilateral hearing loss (MBHL) are being identified. This is based on estimated data from state hearing screening programs that show the prevalences of UHL (0.19/1000 screened) and MBHL $(0.09 / 1000$ screened) are at least half of the expected prevalences for these losses that have been reported in the literature, including a study involving 7 birthing centers. ${ }^{2,3}$ As summarized by Tharpe ${ }^{4}$ in this issue, when left undetected, both UHL and MBHL can adversely affect development and can result in difficulties in speech, language, behavior, and academic achievement in some children. ${ }^{5-22}$ Therefore, this underidentification is potentially of significant concern to audiologists, early interventionists, speech-language pathologists, parents, educators, and others. 
To address the underidentification and the lack of awareness of the significance of UHL and MBHL, the Centers for Disease Control and Prevention Early Hearing Detection and Intervention (EHDI) program and the Marion Downs Hearing Center convened the National Workshop on Mild and Unilateral Hearing Loss in Breckenridge, Colorado, in July 2005. ${ }^{23}$ During this workshop, papers were presented that described the significance of MBHL and UHL, and descriptions of important issues related to the identification and appropriate intervention for children with UHL and MBHL were drafted. This report summarizes the information presented during that workshop regarding screening and diagnosis of UHL and MBHL, as well as the committee recommendations for future research in these areas.

\section{Hearing Screening and Diagnosis}

\section{Background}

As is the case with other degrees of loss, UHL and MBHL do not have established standardized audiologic definitions. At present, the audiologic definitions used for UHL and MBHL vary among countries, states, and providers. These definitions differ based on the audiometric frequencies measured, the tests that are performed (eg, air conduction, bone conduction, and auditory brainstem response [ABR]) to diagnose the loss, or the threshold criteria used to determine the presence or absence of a loss.

For the purposes of the national workshop and this report, the following working definitions of MBHL and UHL were adopted. A permanent MBHL is defined as a calculated or predicted (estimated) airconduction pure tone average (PTA) of 500, 1000, and $2000 \mathrm{~Hz}$ between 20 and $40 \mathrm{~dB}$ hearing level (HL) or air-conduction PTA thresholds greater than $25 \mathrm{~dB}$ HL at 2 or more frequencies above $2000 \mathrm{~Hz}$ (ie, 3000, $4000,6000,8000 \mathrm{~Hz}$ ) in both ears. A permanent UHL is defined as a calculated or predicted air-conduction PTA of 500, 1000, and $2000 \mathrm{~Hz}$ of any level greater than or equal to $20 \mathrm{~dB} \mathrm{HL}$ or air-conduction PTA thresholds greater than $25 \mathrm{~dB} \mathrm{HL}$ at 2 or more frequencies above $2000 \mathrm{~Hz}$ in the affected ear with an air-conduction PTA in the normal hearing ear less than or equal to $15 \mathrm{~dB}$ HL. In both of these definitions, hearing loss exists when there is a permanent air-bone gap of greater than $10 \mathrm{~dB}$ at any 2 of these same frequencies, and medical intervention has been ruled out. Definitions were adapted from Bess et al. ${ }^{22}$
A second challenge has to do with use of the descriptors mild, minimal, and slight, which are used to describe MBHL and UHL and which fail to convey adequately the possible outcomes experienced by affected children. Use of these terms can cause parents to minimize the importance of additional interventions or monitoring or both ${ }^{24}$ and may have the same effect on health care providers and educators. This issue prompted discussion at the national workshop. Although no new terminology was agreed on during the workshop, participants indicated that new terminology is essential to convey the potential effect of these losses and ensure that parents and professionals secure appropriate services for these infants and children.

\section{Newborn Hearing Screening}

During the past decade, the majority of birthing facilities across the United States have adopted universal newborn hearing screening (UNHS) programs. ${ }^{1}$ In conjunction with those screening efforts, states and territories have implemented EHDI programs, ${ }^{1}$ which work to ensure that all children receive hearing screenings, diagnostic audiologic evaluations to confirm losses when indicated by positive screenings, and early intervention services for children with confirmed hearing loss. To help ensure that children receive these services, many EHDI programs are developing tracking and surveillance systems to improve the ability to follow these children by recording screening results and followup actions. In addition, the information in these systems has been used to help calculate aggregate, national-level EHDI statistics. For example, data provided by 48 states and territories for the year 2004 showed that the efforts of EHDI programs had contributed to more than $90 \%$ of newborns being screened at or shortly after birth and the early identification of an increasing number of infants and children with hearing loss within the first few months of life. ${ }^{25,26}$

Although the majority of infants in the United States are now screened, several recent studies report children with less severe degrees of loss, such as UHL and MBHL, are not being identified at the expected prevalence. ${ }^{2,22,27-29}$ As noted in Table 1, state data reported for 2004 indicated that the newborn prevalence of UHL was 0.35/1000 live births screened and 0.16/1000 screened for MBHL, similar to the rates reported by state programs in 2003 . 
Table 1. Prevalence and Prevalence Estimates of UHL and MBHL ${ }^{\mathrm{a}}$

\begin{tabular}{|c|c|c|c|}
\hline $\begin{array}{l}\text { Investigation: Lower Estimate, } \\
\text { Upper Estimate }\end{array}$ & $\begin{array}{l}\text { Population } \\
\text { Screened }\end{array}$ & Age Screened, y & Prevalence Range, per 1000 \\
\hline Bess et $\mathrm{al}^{22}$; Niskar et $\mathrm{al}^{38}$ & UHL & School age (data from literature) & $30.0-56.0$ \\
\hline \multirow[t]{2}{*}{ Dalzell et $\mathrm{al}^{27} ;$ White et $\mathrm{al}^{29}$} & UHL & Newborn (data from literature) & $0.8-2.7$ \\
\hline & UHL & Newborn (data reported by states) & $0.35^{\mathrm{b}}$ \\
\hline Bess et $\mathrm{al}^{22} ;$ Niskar et $\mathrm{al}^{38}$ & MBHL & School age (data from literature) & $10.0-15.0$ \\
\hline \multirow[t]{2}{*}{ Watkin and Baldwin ${ }^{28}$} & MBHL & Newborn (data from literature) & 0.36 \\
\hline & MBHL & Newborn (data reported by states) & $0.16^{\mathrm{b}}$ \\
\hline
\end{tabular}

a. MBHL, mild bilateral hearing loss; UHL, unilateral hearing loss.

b. Based on 2004 Directors of Speech and Hearing Programs in State Health and Welfare Agencies data.

In comparison, research findings in the literature reported prevalence estimates in newborns for UHL that ranged from between $0.8 / 1000$ to $2.7 / 1000 . .^{27,29}$ Only 1 study had published data on the prevalence of MBHL in newborns, reporting a prevalence of $0.36^{28}$ (Table 1). These figures were obtained through a literature review; they represent the lowest and highest prevalence numbers reported in the literature for each respective category. This suggests that the lowest estimated prevalence rates reported in the literature for newborns with UHL and MBHL were at least twice as high as those estimates reported by state and territorial EHDI programs for the year 2004. This difference between newborn prevalence reported by states and that reported in the literature is consistent with findings from 2 population-based studies that suggest permanent UHL and MBHL in infants and children typically remain undetected unless special efforts are taken to identify these losses. ${ }^{2,22}$

Furthermore, the overall prevalence rates for all types of hearing loss in newborns, including UHL and MBHL reported by state and territorial EHDI programs, have historically been lower than the prevalence rates reported in the literature. This can be explained in part by the following factors. Some children with hearing loss might not be identified because existing screening protocols are not sensitive enough to identify hearing loss less than $30 \mathrm{~dB}$. Also, estimates indicate that approximately $50 \%$ of results of recommended follow-up tests of infants who do not pass the newborn hearing screening are not reported. In 2004, the overall prevalence for all types and degrees of hearing loss reported by EHDI programs was $1.1 / 1000$ infants screened, which is at the low range of the 1 to $3 / 1000$ cases of hearing loss published in the literature. ${ }^{30,31}$ As a result, it is expected that the rates of UHL and MBHL reported by EHDI programs would also be low relative to published prevalence rates.

In addition, published research is largely based on controlled studies in clinics or hospitals, in which cases of UHL and MBHL are more likely to be identified. This is because parents or guardians are more likely to take their children to a clinic when they show symptoms of hearing loss as compared with prevalence in an unbiased population-based study. Conversely, a majority of EHDI programs rely on reports from audiologists and physicians to determine the number, type, and severity of all cases of hearing loss in a given year. Given that this reporting is largely voluntary, it is possible that not all cases of identified hearing loss, including UHL and MBHL, are reported. This could reduce the number of cases of hearing loss being reported to an EHDI program, including cases of UHL and MBHL.

\section{Newborn Hearing Screening Technology}

There are multiple issues surrounding the usefulness and application of existing forms of screening technologies commonly used for UNHS in the identification of mild forms of hearing loss in 1 or both ears. According to the Joint Committee on Infant Hearing (2000) position statement, "depending on the screening technology selected, infants with hearing loss $<30$ $\mathrm{dB}$ or with hearing loss related to auditory neuropathy or neural conduction disorders may not be detected in a UNHS program." ${ }^{32}$ Few data exist regarding the effectiveness of current screening devices for use in early identification of mild UHL and MBHL. One study $^{2}$ concluded that an existing newborn screening 
protocol that resulted in a "pass" outcome (otoacoustic emission [OAE] fail followed by automated $\mathrm{ABR}[\mathrm{A}-\mathrm{ABR}]$ pass) might have missed up to $70 \%$ of all cases of mild unilateral and bilateral hearing loss of approximately 25 to $30 \mathrm{~dB}$. In this study, 1524 of 86634 infants screened at 7 birthing centers that used a 2-stage OAE/A-ABR screening protocol were enrolled. Criteria for enrollment included failing the OAE screening but passing the A-ABR screening. When these infants were a mean 9.7 months of age, diagnostic audiologic testing using visual reinforcement audiometry was performed for 973 infants.

However, a modification of the screening technology (eg, lowering the stimulus level from $35 \mathrm{~dB}$ normal HL [nHL] to $25 \mathrm{~dB}$ nHL in A-ABR screening devices) is not a simple solution. Such a modification would likely result in a significant increase in the number of false-positive cases. That situation would undoubtedly burden EHDI programs with substantially increased follow-up costs and place extra responsibility on an already overburdened system. This, in turn, could potentially undermine confidence in the EHDI process. ${ }^{33}$

Furthermore, no national or international standards currently exist for the calibration of OAE or A-ABR screening devices. Only 1 international standard (International Electrical Contractors No. 60645-3) is available for the calibration of stimuli (such as clicks used in screening ABR devices). It is not clear that manufacturers of screening devices used in the newborn period calibrate their instruments using a uniform standard. Moreover, screening algorithms and pass-refer criteria vary among manufacturers. Therefore, the screening outcome obtained with one OAE screening instrument might not yield the same outcome (pass-refer) as a device from another manufacturer. ${ }^{34}$

Stimuli used for evoking OAEs are presented at a known sound pressure level that is calibrated in the ear canal of an individual infant or child. However, the level of the A-ABR clicks at the tympanic membrane varies to some degree depending on the type of earphone (ie, supra-aural or insert) and the size of the infant's ear canal. Thus, the actual level of the test signal can vary appreciably among infants regardless of the audiometric HL ( $\mathrm{dB} \mathrm{nHL}$ ) referenced as the pass-fail criterion level by the manufacturer. ${ }^{35}$ That is, the intensity of the sound presented at the eardrums of various infants can be different. Finally, there are limited data available on specific devices used by newborn hearing screening programs that allow program administrators or audiologists to appreciate the performance characteristics of pass-refer algorithms.

\section{Preschool and School-aged Screening}

One possible solution to identifying children with UHL and MBHL who were missed during the newborn period or who developed a hearing loss after this time is to provide a universal hearing screening in early childhood. However, after the newborn hearing screening and before starting school, there is no common event that currently exists to trigger a second hearing screening for young children. The American Academy of Pediatrics, in a 2006 position statement, ${ }^{36}$ recommended that developmental screening tests be administered at a child's 30-month visit. This potentially presents a good opportunity for universal preschool hearing screening. However, because the 30 -month visit is often not reimbursable by thirdparty payers, it is unknown how many families would be unable to comply with this recommendation because of financial issues. In addition, objective hearing screening devices (eg, OAE screeners) are not available in the majority of pediatricians' offices, and at 30 months, valid behavioral screening of toddlers is unlikely to be feasible in pediatric practice.

The importance of early identification of hearing loss, including MBHL and UHL, might be compromised by a lack of standardized hearing screening programs in the United States in the toddler and preschool periods. Currently, a good opportunity for an additional universal hearing screening beyond the newborn period is during entry into school. However, although $96 \%$ of children enroll in the US public school system at 5 to 6 years of age, ${ }^{37}$ the acoustic environments in schools for an entry screen and any subsequent hearing screens are often inadequate for obtaining accurate results. ${ }^{22}$

Prevalence estimates of hearing loss in school-aged children (approximately 6-19 years of age) range from $10 / 1000$ to $15 / 1000$ for MBHL and 30/1000 to $56 / 1000$ for UHL. ${ }^{22,38}$ This variance in prevalence estimates is likely the result of factors similar to those found with screening newborns, as well as the inclusion of children with progressive or late-onset hearing loss. Causes of progressive or late-onset hearing loss include genetic and acquired environmental factors, such as teratogenic agents, infections, illnesses, trauma, and noise-induced hearing loss. Consequently, prevalence rates appear to increase from the newborn period to age at entry to school. 
Behavioral audiometric and tympanometric screening tests applied at school age are intended to identify temporary as well as permanent hearing loss, including mild degrees of hearing loss in 1 or both ears. Yet as previously noted, the acoustic conditions under which school-based identification programs are administered, as well as screening pass-fail criteria, are not always conducive to achieving those goals. To date, there are few studies that have examined the effectiveness of physiologic technologies, such as OAE, or behavioral techniques in preschool and school-aged screening programs. ${ }^{39}$

\section{Research Needs in Screening for UHL and MBHL}

One of the most important questions to answer at this point is when we should attempt to identify these losses: in the newborn, preschool, or school-aged period? This question will be answered, in part, by our ability to intervene with these age groups. That is, do we have proven, effective interventions for infants, toddlers, preschoolers, and school-aged children with MBHL and UHL? This question is addressed in another article in this issue that focuses on management of children with UHL and MBHL. ${ }^{40}$ Until we can answer these questions, it is reasonable to focus some attention on the feasibility of screening for these losses. This would include ways to detect mild hearing loss in 1 or both ears without increasing falsepositive rates (eg, modify or develop new screening technologies). Specifically, an option for pass-refer algorithms designed to detect these degrees of hearing loss should be considered as an option on screening devices. Cost-effectiveness analysis of these modifications in screening technology would be necessary, including the determination of false-positive and false-negative outcomes.

It was considered important by those involved in the July 2005 national workshop that studies be carried out in typical, real-world environments. This is especially important for preschool and school-aged screenings that are typically conducted in less-thanoptimum acoustic environments and, often, by those who know little about acoustics and hearing. Such screening methodologies might include language and functional communication tools used in conjunction with technology (such as hearing aids or frequency modulated systems). Finally, prevalence studies of UHL and MBHL in childhood (across ages) are needed to assist in making management decisions (such as the use of hearing technology, enrollment in speech-language pathology, or special support services in the school).

\section{Diagnostic Evaluation}

The differentiation of mild hearing loss from normal hearing is also challenging using the battery of audiologic test procedures available for use in a comprehensive audiologic assessment of infants and children at risk for hearing loss. The battery is sufficiently sensitive and specific to differentiate normal hearing from hearing loss of mild to profound degree. However, the differentiation of minimal hearing loss from normal hearing can be challenging in the period when only electrophysiological and physiological tests are available. Unlike hearing screenings, diagnostic audiologic tests are typically conducted in acoustically controlled environments with a wide range of available tests that can accommodate children at different levels of development. A battery of tests consisting of electrophysiological, physiological, and behavioral test methods can be used for delineating among sensory, neural, conductive, and mixed types of hearing losses. Differentiating among these forms of hearing loss is critical for identifying all degrees of mild permanent hearing loss from temporary conductive loss associated with otitis media with effusion, which is particularly prevalent in infancy and early childhood and can compromise identification of permanent hearing loss. ${ }^{41,42}$

When accurate and timely audiologic information is available after referral from a hearing screening program, optimal amplification and other appropriate interventions can be provided. Unfortunately, there can be a lack of audiologic facilities, especially in rural parts of the country, that can provide all of the requisite tests and meet the differential diagnostic and amplification fitting needs of this pediatric population. ${ }^{43}$ In addition, a shortage of audiologists who specialize in serving infants and young children limits the availability in some areas of both early and comprehensive audiologic assessment, as well as amplification selection and fitting. This can lead to a delay in diagnosis and intervention for some children. This initial delay can reduce the potential effectiveness of other intervention efforts and can result in frustration for families seeking services that are unavailable in their communities. These factors might be especially relevant in the quest to diagnose and manage mild permanent hearing loss. ${ }^{41}$ 
Part of the difficulty in securing qualified audiologists who can serve infants and young children might lie with audiology training programs. In 2 separate surveys of training programs within the United States conducted in 1987 and again in 2000, ${ }^{44,45}$ audiologists were found to receive insufficient academic and clinical preparation for the appropriate provision of either diagnostic or intervention services to infants and children. This situation has likely not changed appreciably with the change in entrylevel degree to the doctor of audiology.

In keeping with these concerns, there was much discussion during the national workshop about the establishment of centers of expertise that could offer multidisciplinary services, including comprehensive, state-of-the-art audiology services to infants and children who are deaf and hard of hearing, including those with UHL and MBHL, and their families. Such centers of expertise were also considered important for the selection and fitting of appropriate amplification to children with UHL and MBHL.

Just as with screening for MBHL, there are some equipment limitations in the diagnostic process as well. Audiologic evaluation should include electrophysiological, physiological, and age-appropriate behavioral test methods. ${ }^{46}$ In the diagnostic assessment, as well as in the selection and fitting of amplification, the acoustic characteristics of the child's external ear and the sound pressure level in the infant's occluded ear should be considered in the determination of hearing sensitivity. ${ }^{47}$ In addition, the fact that $\mathrm{dB} H \mathrm{HL}$ and $\mathrm{dB}$ nHL are referenced to average adult ear canal acoustics contributes to the differences one may observe when estimating thresholds in infants and young children. At this time, however, the clinical use of correction factors for both visual reinforcement audiometry and diagnostic ABR procedures is inconsistent. That is, there might be differences across and within facilities in the actual sound pressure levels (expressed audiometrically or electrophysiologically as $\mathrm{dB} \mathrm{HL}, \mathrm{dB}$ nHL, or dB estimated HL) that are delivered to the infant's or child's ear. These measured thresholds are ultimately used to differentiate normal hearing from mild hearing loss, to determine the degree of hearing loss in the affected ear of a child with UHL, or to monitor the stability of the hearing loss over time. Audiologic evaluation with behavioral or ABR diagnostic procedures should also include bone conduction testing whenever feasible.

There are additional challenges using tympanometry to determine the type of hearing loss (conductive, sensory, neural, or mixed) in infants. The accurate detection of otitis media with effusion in infants younger than approximately 4 months requires the use of a high-frequency $(1000-\mathrm{Hz})$ probe tone. ${ }^{48,49}$ However, this option is not available on all clinical immittance instruments used with infants. Finally, additional data are needed to determine the usefulness of the auditory steady-state response in the pediatric audiologic diagnostic test protocol for use in determining the type, degree, and configuration of a young child's hearing loss. Currently, auditory steadystate response yields highly variable results in lowfrequency regions and at low intensity levels, the latter being particularly problematic for diagnosing cases of mild hearing loss. ${ }^{46}$

\section{Research Needs in the Diagnosis of UHL and MBHL}

Numerous research needs were identified by the attendees of the national workshop. These included, among others, the need to determine the natural history of UHL, including the frequency of progression from UHL to bilateral hearing loss and the probability of progression of mild hearing loss to a more severe degree of hearing loss. This information would be useful in determining an audiologic monitoring schedule for children identified with UHL. Additional normative data are also needed for airand bone-conduction thresholds (behavioral and $\mathrm{ABR}$ ) for infants with normal hearing to assist in delineating between those infants with normal hearing and those with MBHL. It is also unclear which audiologic tests or combination of tests is most likely to differentiate mild UHL and MBHL from normal hearing. Finally, it would be of interest to determine the diagnostic yield of UHL and MBHL from other medical tests such as imaging studies, laboratory testing for cytomegalovirus and other teratogens, and genetic testing for the presence of known mutations causing hearing loss (eg, connexin 26).

It is apparent from the studies conducted thus far on the identification and diagnosis of UHL and MBHL in infants and young children that there is much clinical research work to be accomplished in this area. At present, we are not identifying many of these children in the newborn period and are likely missing many of them through school-based hearing screening efforts, although data on the latter are lacking. We have yet to determine the optimal time at which to identify and intervene with these children or the optimal measures to assist us in their identification. 
In fact, we have barely scratched the surface on what needs to be done with these children to avert developmental problems once we do identify them. We hope that the outcomes of the national workshop represent a contribution to this ongoing effort.

\section{Acknowledgment}

The preparers of this article acknowledge the valuable contributions of the following people for their time, effort, and commitment that guided the content and development of this document: Vickie Thomson, Marion Downs Hearing Center; Arlene StredlerBrown, Marion Downs Hearing Center; Lynn Luethke, National Institutes for Health; Anne Marie Tharpe, Vanderbilt University; Sharon Ringwalt, National Early Childhood Technical Assistance Center; Janet Farrell, Massachusetts Department of Public Health; John Eichwald, Centers for Disease Control and Prevention; Jamie Elliot, Centers for Disease Control and Prevention.

\section{References}

1. Gaffney M, Gamble M, Costa P, Holstrum J, Boyle C. Infants tested for hearing loss: United States, 1999-2001. MMWR Morb Mortal Wkly Rep. 2003;52:981-984.

2. Johnson JL, White KR, Widen JE, et al. A multicenter evaluation of how many infants with permanent hearing loss pass a two-stage otoacoustic emissions/automated auditory brainstem response newborn hearing screening protocol. Pediatrics. 2005;1 16:663-672.

3. Gravel J. Prevalence and Screening in Newborns. National Workshop on Mild and Unilateral Hearing Loss: Workshop Proceedings 2005. Breckenridge, CO: Centers for Disease Control and Prevention; 2005:15-7.

4. Tharpe AM. Unilateral and mild bilateral hearing loss in children: past and current perspectives. Trends Amplif. 2008;12: 7-15.

5. Yoshinaga Itano C, Sedey AL, Coulter BA, Mehl AL. Language of early- and later-identified children with hearing loss. Pediatrics. 1998;102:1161-1171.

6. Wake M, Hughes EK, Poulakis Z, Collins C, Rickards FW. Outcomes of children with mild-profound congenital hearing loss at 7 to 8 years: a population study. Ear Hear. 2004;25:1-8.

7. Wake M, Poulakis Z. Slight and mild hearing loss in primary school children. J Paediatr Child Health. 2004; 40:11-13.

8. Ruscetta MN, Arjmand EM, Pratt SR. Speech recognition abilities in noise for children with severe-toprofound unilateral hearing impairment. Int J Pediatr Otorhinolaryngol. 2005;69:771-779.
9. Oyler RF, Oyler AL, Matkin ND. Unilateral hearing loss: demographics and educational impact. Lang Speech Hear Serv Sch. 1988;19:191-210.

10. Oyler RF, Oyler AL, Matkin ND. Warning: a unilateral hearing loss may be detrimental to a child's academic career. Hear J. 1987;9:18-22.

11. Moeller MP. Early intervention and language development in children who are deaf and hard of hearing. Pediatrics. 2000;106:1-9.

12. Lieu JE. Speech-language and educational consequences of unilateral hearing loss in children. Arch Otolaryngol Head Neck Surg. 2004;130:524-530.

13. Klee TM, Davis-Dansky E. A comparison of unilaterally hearing-impaired children and normal-hearing children on a battery of standardized language tests. Ear Hear. 1986;7:27-37.

14. Kiese-Himmel C, Ohlwein S. Characteristics of children with permanent mild hearing impairment. Folia Phoniatr Logop. 2003;55:70-79.

15. Davis JM, Elfenbein J, Schum R, Bentler RA. Effects of mild and moderate hearing impairments on language, educational, and psychosocial behavior of children. J Speech Hear Disord. 1986;51:53-62.

16. Davis A, Reeve K, Hind S, Bamford J. Children With Mild and Unilateral Hearing Loss: A Sound Foundation Through Early Amplification 2001. Proceedings of the Second International Conference, Section V. Stafa, Switzerland: Phonak; 2001:179-186.

17. Brookhouser PE, Auslander MC, Meskan ME. The pattern and stability of postmeningitic hearing loss in children. Laryngoscope. 1988;98:940-948.

18. Brookhouser PE, Worthington DW, Kelly WJ. Unilateral hearing loss in children. Laryngoscope. 1991;101(12 pt 1):1264-1272.

19. Blair JC, Peterson ME, Viehwed SH. The effects of mild sensorineuroal hearing loss on academic performance of young school-age children. Volta Rev. 1985;87:87-93.

20. Bess FH, Tharpe AM. Case history data on unilaterally hearing-impaired children. Ear Hear. 1986;7:14-19.

21. Bess FH, Tharpe AM. Performance and management of children with unilateral sensorineural hearing loss. Scand Audiol Suppl. 1988;30:75-79.

22. Bess FH, Dodd-Murphy J, Parker RA. Children with minimal sensorineural hearing loss: prevalence, educational performance, and functional status. Ear Hear. 1998;19:339-354.

23. Proceedings from the July 2005 National Workshop on Mild and Unilateral Hearing Loss 2005. Available at: http://www.cdc.gov/ncbddd/ehdi/documents/unilateralhl/ Mild_Uni_2005\%20Workshop_Proceedings.pdf. Accessed March 8, 2007.

24. Haggard RS, Primus MA. Parental perceptions of hearing loss classification in children. Am J Audiol. 1999;8:83-92.

25. Directors of Speech and Hearing Programs in State Health and Welfare Agencies. Estimated number of 
infants screened for hearing loss: 2003. Available at: http://www.cdc.gov/ncbddd/ehdi/2003/Screen_03_web_ D.pdf. Accessed March 5, 2007.

26. Norton SJ, Gorga MP, Widen JE, et al. Identification of neonatal hearing impairment: evaluation of transient evoked otoacoustic emission, distortion product otoacoustic emission, and auditory brain stem response test performance. Ear Hear. 2000;2 1:508-528.

27. Dalzell L, Orlando M, MacDonald M, et al. The New York State Universal Newborn Hearing Screening Demonstration Project: ages of hearing loss identification, hearing aid fitting, and enrollment in early intervention. Ear Hear. 2000;21:118-130.

28. Watkin PM, Baldwin M. Confirmation of deafness in infancy. Arch Dis Child. 1999;81:380-389.

29. White KR, Vohr BR, Maxon AB, Behrens TR, McPherson MG, Mauk GW. Screening all newborns for hearing loss using transient evoked otoacoustic emissions. Int J Pediatr Otorhinolaryngol. 1994;29:203-217.

30. Finitzo T, Albright K, O'Neal J. The newborn with hearing loss: detection in the nursery. Pediatrics. 1998;102: 1452-1460.

31. Van Naarden K, Decouflé P, Caldwell K. Prevalence and characteristics of children with serious hearing impairment in metropolitan Atlanta: 1991-1993. Pediatrics. 1999;103:570-575.

32. Joint Committee on Infant Hearing, American Academy of Audiology, American Academy of Pediatrics, American Speech-Language-Hearing Association, Directors of Speech and Hearing Programs in State Health and Welfare Agencies. Year 2000 position statement: principles and guidelines for early hearing detection and intervention programs. Pediatrics. 2000;106:798-817.

33. Gravel JS, White KR, Johnson JL, et al. A multisite study to examine the efficacy of the otoacoustic emission/ automated auditory brainstem response newborn hearing screening protocol: recommendations for policy, practice, and research. Am J Audiol. 2005;14: S217-S228.

34. Barker SE, Lesperance MM, Kileny PR. Outcome of newborn hearing screening by ABR compared with four different DPOAE pass criteria. Am J Audiol. 2000;9: 142-148.

35. Stevens J, Wood S. NHSP in England: screening equipment. Paper presented at: International Newborn Hearing Screening Symposium; May 2004; Como, Italy.

36. American Academy of Pediatrics- Council on Children With Disabilities, Section on Developmental Behavioral Pediatrics, Bright Futures Steering Committee and Medical Home Initiatives for Children With Special Needs Project Advisory Committee. Identifying infants and young children with developmental disorders in the medical home: an algorithm for developmental surveillance and screening (policy statement). Pediatrics. 2006; 118:405-420.

37. Jamison A, Martinez GM, Curry AE. School Enrollment in the United States: Social and Economic Characteristics of Students, October 1999 [current population reports]. Washington, DC: US Census Bureau; 1999. Available at: http://www.census.gov/population/www/socdemo/school. html. Accessed March 29, 2007.

38. Niskar AS, Kieszak SM, Holmes A, Esteban E, Rubin C, Brody DJ. Prevalence of hearing loss among children 6 to 19 years of age. J Am Med Assoc. 1998;279:1071-1075.

39. Nozza RJ, Sabo DL, Mandel EM. A role for otoacoustic emissions in screening for hearing impairment and middle ear disorders in school-age children. Ear Hear. 1997;18:227-239.

40. Gaffney M, Ross DS, Holstrum J, Oyler RF, Gravel J. Early intervention for children with unilateral and mild bilateral degrees of hearing loss. Trends Amplif. 2008; 12:35-41.

41. Gravel JS. Potential pitfalls in the audiological assessment of infants and young children. In: Seewald RC, Gravel JS, eds. A Sound Foundation Through Early Amplification. Stafa, Switzerland: Phonak AG; 2002:85-102.

42. Stapells DR, Ruben RJ. Auditory brain stem responses to bone-conducted tones in infants. Ann Otol Rhinol Laryngol. 1989;98(12 pt 1):941-949.

43. White KR. The current status of EHDI programs in the United States. Ment Retard Dev Disabil Res Rev. 2003;9:79-88.

44. Oyler RF, Matkin ND. National survey of educational preparation in pediatric audiology. Am Speech Lang Hear Assoc. 1987;29:27-33.

45. Oyler RF, Gross SR. Survey of educational preparation in pediatric audiology: a decade later. Commun Dis Quart. 2000;21:195-209.

46. American Speech Language Hearing Association. Guidelines for the audiological assessment of children from birth to five years of age, 2004. Available at: http://www.asha.org/NR/rdonlyres/0BB7C840-27D24DC6-861B-1709ADD78BAF/ 0/v2GLAudAssess Child. pdf. Accessed March 5, 2007.

47. American Academy of Audiology. Pediatric amplification guidelines, 2003. Available at: http://www.audiology.org/ professional/positions/pedamp.pdf. Accessed March 5, 2007.

48. Margolis RH, Bass-Ringdahl S, Hanks WD, Holte L, Zapala DA. Tympanometry in newborn infants: $1 \mathrm{kHz}$ norms. J Am Acad Audiol. 2003;14:383-392.

49. Kei J, Allison-Levick J, Dockray J, et al. High-frequency $(1000 \mathrm{~Hz})$ tympanometry in normal neonates. J Am Acad Audiol. 2003;14:20-28. 